\title{
Modified walnut shell filter material for the enhanced removal of oil from oilfield wastewater
}

\author{
Xianqing Yin ${ }^{1}$, Jian Zhang ${ }^{2,3}$, Xiujun Wang ${ }^{2,3}$, Mijia $\mathrm{Zhu}^{{ }^{+}}$ \\ ${ }^{1}$ State Key Laboratory of Petroleum Pollution Control (Yangtze University), School of Chemistry and Environmental Engineering, Yangtze University, \\ Jingzhou, Hubei 434023, China \\ ${ }^{2}$ State Key Laboratory of Offshore Oil Exploitation, Beijing 100027, China \\ ${ }^{3}$ CNOOC Research Institute, Beijing 100027, China
}

\section{ABSTRACT}

While polymer oil recovery greatly improves oil recovery, the polymer injected into the formation undergoes shearing, degradation, etc., and returns with the output liquid in the form of an anionic polyacrylamide of lower molecular weight, resulting in a production fluid. There is residual polymer, and this kind of polymer-containing wastewater is easy to contaminate the walnut shell filter in the sewage treatment process, resulting in failure of the walnut shell filter material, introduce hydrophilic sulfonic acid groups on the surface of the walnut shell filter material to lipophilic to hydrophilic of the surface. The dosage of $\mathrm{NaHSO}_{3}$ is $10-20 \%$ of the mass fraction of walnut shell filter (mass ratio \%). Reflux reaction, and the reaction time was $1-5 \mathrm{~h}$; the surface wettability of the walnut shell reversed. The surface contact angle drops from $95^{\circ}$ before modification to $36.75^{\circ}-66.25^{\circ}$, Its surface hydrophilic oleophobic performance has been significantly improved, Treatment of $150-230 \mathrm{mg} / \mathrm{L}$ of oily sewage, filtration and oil removal rate increased from $38.05 \%$ to $89.51 \%$ by modification; Backwashing and degreasing effect is increased by 4 times.

Keywords: Filter material, Modified walnut shell, Oil removal rate, Sulphite, Surface contact angle

\section{Introduction}

While polymer oil recovery greatly improves oil recovery, the polymer injected into the formation undergoes shearing, degradation, etc., and returns with the output liquid in the form of an anionic polyacrylamide of lower molecular weight, resulting in a production fluid [1]. This kind of polymer-containing wastewater is easy to contaminate the walnut shell filter in the treatment process, which leads to the walnut shell not being regenerated by backwashing, the failure of the knot is lost and the filtration is lost, and the service life is shortened [2].

Filter materials in the oily wastewater treatment of oilfields include quartz sand, walnut shell, microporous ceramic, membrane and fibre medium, different filter media are affected by oil contamination to a greater extent. The walnut shell can easily produce hydraulic rebound without considerable backwashing strength due to its wide range of sources, strong adsorption, interception capability, anti-oil immersion, high hardness, good abrasion resistance, stable chemical properties and low particle density. The walnut shell can be repeatedly used in many times to restore the adsorption and degreasing effect, and it is widely utilised as the filter material in oilfield filters [3-5]. After this material is used to treat polymercontaining oily wastewater for a period, the knotting phenomenon is likely to occur. The effect of backwashing and degreasing is drastically reduced, and the degreasing rate is shortened.

Here, in order to realize the low-cost and effective filtration separation and treatment of the polymer-containing oily wastewater, the surface of the hydrophilic and oleophobic walnut shell filter material is prepared by a simple chemical modification method. In this study, a hydrophilic sulfonic acid group is introduced on the surface of the walnut shell and chemically bonded to the surface functional group of the walnut shell, a stable coating layer is formed on the surface of the walnut shell, the micropores become smaller, the surface is more dense, the surface wettability is greatly improved, and the super-hydrophilic and underwater oleophobic, and the compressive strength does not change. What
This is an Open Access article distributed under the terms of the Creative Commons Attribution Non-Commercial License (http://creativecommons.org/licenses/by-nc/3.0/) which permits unrestricted non-commercial use, distribution, and reproduction in any medium, provided the original work is properly cited.

Copyright (C) 2021 Korean Society of Environmental Engineers
Received September 03, 2019 Accepted February 11, 2020

${ }^{\dagger}$ Corresponding author

Email: zhumijia128@163.com

Tel: +86-716-8060472 Fax: +86-716-8060472

ORCID: 0000-0003-0061-8305 
is important is that the filtration degreasing rate is greatly improved. Filter material backwashing and oil removal is easy, prolonging the service life of walnut shell filter material. Therefore, we expect that the modified walnut shell has a wide range of potential applications in the treatment of polymer-containing oily wastewater.

\section{Material and Methods}

\subsection{Materials}

Industrial walnut shell filter used in the field was obtained from Gongyi Jingyu Filter Material Company. The walnut shell filter material with a particle size of 1.6-2.0 mm was washed with deionised water until the water was clear, and then it was dried at $105^{\circ} \mathrm{C}$ for $3 \mathrm{~h}$. Industrial products $\mathrm{Na}_{2} \mathrm{CO}_{3}, \mathrm{NaHSO}_{3}$ and $\mathrm{Na}_{2} \mathrm{SO}_{3}$ from Hunan Zhongcheng Chemical Co.,Ltd, The polymer AP-P4 (relative molecular mass of 10 million, hydrolysis degree of $19 \%$, and hydrophobic base content of $0.1 \%$ ) is water-soluble hydrophobically associated polyacrylamide, from Sichuan Guangya Technology Co., Ltd. Crude oil (API value was $13.9,20^{\circ} \mathrm{C}$ density was $0.9701 \mathrm{~g} / \mathrm{cm}^{3}$, colloid content was $21.8 \%$, paraffin content was $1.31 \%$, and n-pentane insoluble matter was $9.94 \%$ ) form the target oilfield provided the $1,000 \mathrm{~mL}$ three-neck glass reaction bottle device, including stirring apparatus, thermostatic oil bath, condensing reflux unit and thermometer.

\subsection{Instrument}

DSA30 contact angle measuring instrument (KRUSS), MIRA3 scanning electron microscope (Tescan), M630 NIR spectrometer (Bruker), TU-1810PC UV-Vis spectrophotometer (Beijing General Analysis), WAW-1000B universal pressure testing machine (Jinan Tianhua), GF30 high shear emulsifier (Fluko), Self-made CH-II micro-continuous sewage treatment unit and filter $(\mathrm{DN}=40 \mathrm{~mm}$, $\mathrm{H}=400 \mathrm{~mm})$.

\subsection{Polymer-containing Oily Wastewater Sample}

The experimental water sample was obtained from an oilfield on-site wastewater with a salinity of $9374.13 \mathrm{mg} / \mathrm{L}$. The mass concentration of the polymer simulating the water was $50 \mathrm{mg} / \mathrm{L}$. Then, the water sample added with the polymer was heated at $60^{\circ} \mathrm{C}$, added with the standard crude oil of the oilfield and emulsified by a high shear emulsifier at $12,600 \mathrm{r} / \mathrm{min}$ for $5.00-10.00 \mathrm{~min}$ to obtain a water sample surface free from oil slick; its oil content was 150.00 $230.00 \mathrm{mg} / \mathrm{L}$. The water sample oil content was determined in accordance with the 'determination of oil content in oilfield waste- water-spectrophotometric method' (SY/T 0530-2011).

\subsection{Surface Modification of Filter Material}

A certain amount of walnut shell filter material $m_{1}$, adding different amounts of ionized water, then adding three kinds of reaction agent $m_{\mathrm{x}}$ (mass ratio $\mathrm{m}_{\mathrm{x}} / \mathrm{m}_{1} \%$ ), reacting at different temperatures for different time, the series reaction conditions are shown in Table 1.

A certain amount of washed walnut shell filter material is added to the reaction bottle provided with the stirring device and the condenser, and deionized water is added until the filter material is completely immersed in a certain ratio of chemical solution under reflux for different time. After the reaction is completed, the filter material was poured out, rinsed with clear water until colorless and dried in an oven at $105^{\circ} \mathrm{C}$ for $3 \mathrm{~h}$. The unmodified filter material is No. 0 , and the reaction conditions are changed to obtain a series of modified filter materials No. 1-6 of different reaction conditions.

\subsection{Characterisation of Filter Material}

The surface morphologies of No. 0 unmodified and No. 3 modified filter materials were analysed via scanning electron microscopy (SEM). Treatment process: spray-gold treatment to enhance the conductivity of the filter material. Test condition: $15.0 \mathrm{kV}$. The types and contents of the surface elements of the filter materials were analysed via energy-dispersive X-ray spectroscopy (EDS) and XPS, respectively.

\subsection{Determination of the Surface Wettability of Filter Materials}

The surface contact angle of the series of surface modified and unmodified filter materials was determined using the sitting drop method, and the diameter of the bayonet glue needle was 0.247 $\mathrm{mm}$.

\subsection{Filter Material Compression Resistance Test}

The steel pipe with an inner diameter of $40 \mathrm{~mm}$ was filled with walnut shells pressed using a steel piston. The test machine started the compression resistance test by slowly pressurising the filter material with a force speed of $0.10 \mathrm{kN} / \mathrm{s}$ to obtain the stress-deformation curve before and after modification.

\subsection{Filter Structure and Filter Material Filling Method}

Filter packing: DN400, Filter was filled with $360.00 \mathrm{~mm}$ of Walnut shells, the structure of which is shown in Fig. 1.

Table 1. The Dosage and Reaction Conditions of the Filter Materials

\begin{tabular}{lccccccc}
\hline Serial number & Walnut shell/g & Deionized water/g & NAC/\% & YNS/\% & NHS/\% & Temperature/ ${ }^{\circ} \mathbf{C}$ & Reaction time/h \\
\hline 1 & 250 & 250 & 10 & 10 & 0.10 & 95 & 1 \\
2 & 250 & 250 & 10 & 5 & 0.15 & 5 & 105 \\
3 & 250 & 250 & 20 & 10 & 0.10 & 105 & 3 \\
4 & 200 & 240 & 10 & 5 & 0.10 & 105 & 3 \\
5 & 200 & 240 & 10 & 10 & 0.15 & 100 & 3 \\
6 & 200 & 240 & 5 & 5 & 0.20 & 105 & 3 \\
\hline
\end{tabular}



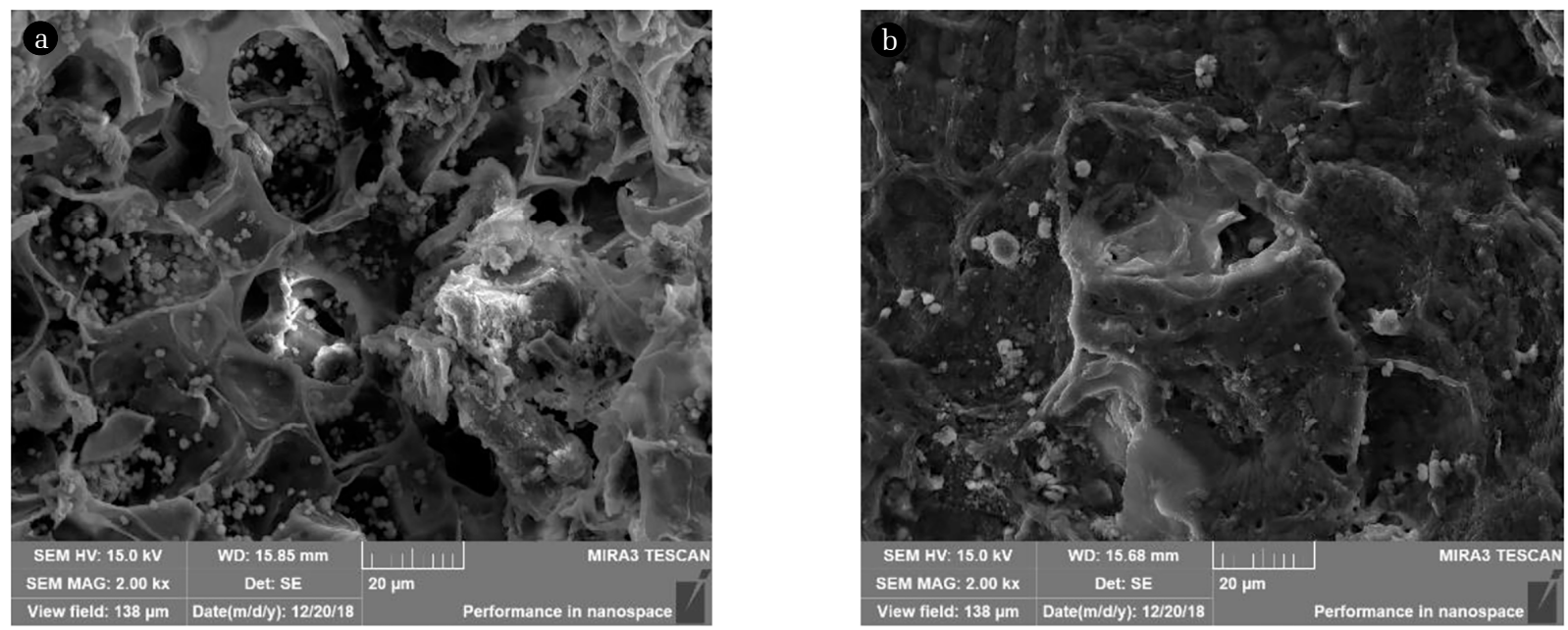

Fig. 1. (a) SEM image of oral walnut shell and (b) Modified walnut shell.

\subsection{Continuous Dynamic Degreasing Experiment with Different Filter Materials}

The unfiltered filter material No. 0, modified filter material No. 1 and modified filter material No. 3 were used to carry out the continuous filter dynamic degreasing experiment. The filling methods of the five kinds of walnut shell filter materials are shown in Table 2.

The sewage $\left(60^{\circ} \mathrm{C}\right)$ is continuously into the filter by the pump, and the filter material is completely immersed in the water during the filtration process, and the pump is set to stabilize the same filtration speed for the filtration experiment. Each time the $3 \mathrm{~L}$ water sample of the filter outlet is collected, total of 5 filter batches.

Table 2. Filter and Filter Material Filling Method

\begin{tabular}{cc}
\hline Filte filling Item & Filter material filling method \\
\hline Filter a & 0\# filter material \\
Filter b & 1\# filter material \\
Filter c & $3 \#$ filter material \\
Filter d & $3 \#$ and $0 \#$ filter materials $1: 1(\mathrm{~m} / \mathrm{m})$ \\
Filtre e & $1 \#$ and $0 \#$ filter materials $1: 1(\mathrm{~m} / \mathrm{m})$ \\
\hline
\end{tabular}

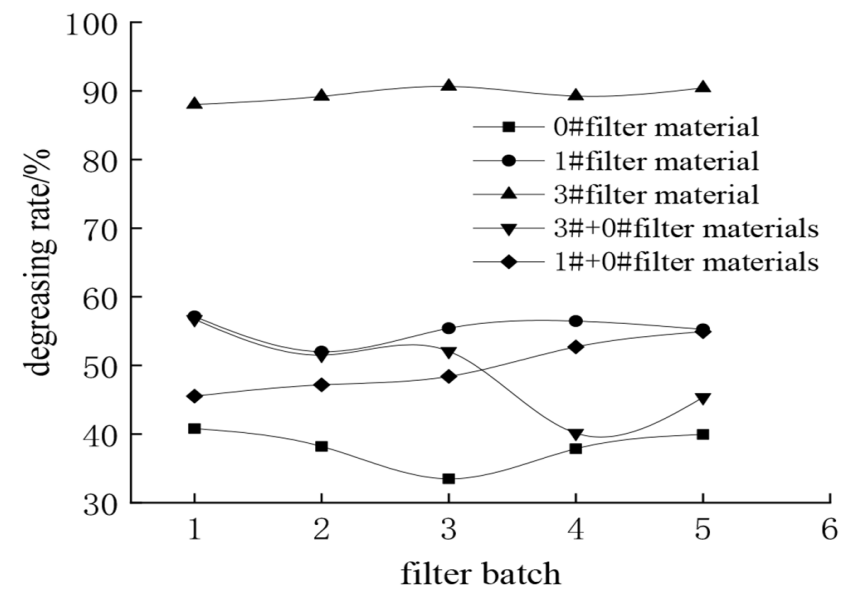

Fig. 2. Comparison of oil removal rate of different filled filter materials.

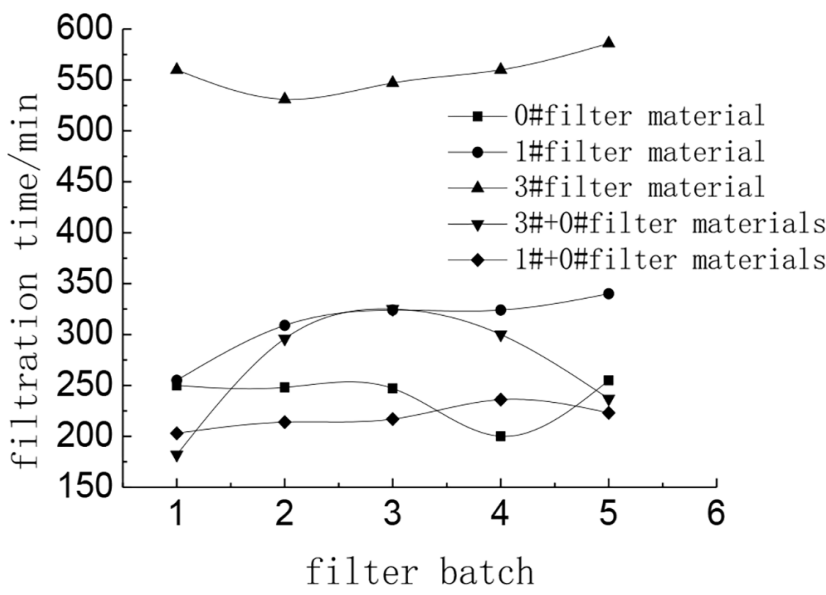

Fig. 3. Comparison of filtration time for filtering the same amount of water.

Record the filtration time of five filtered batches and analyze the oil content of the filtered water sample. After each batch of filter material is filtered, the same conditions as the filter pump are used to pump the $2 \mathrm{~L}\left(60^{\circ} \mathrm{C}\right)$ field sewage from the lower end of the filter to reverse backwash the filter material. Collect the backwash water sample at the outlet at the top of the filter to measure the oil content. The trend of the experimental degreasing rate and filtration time of the filter materials $a$ to $e$ filled with different filter materials is shown in Fig. 2 and Fig. 3.

\section{Results and Discussion}

\subsection{Optimum Conditions for Filter Material Modification}

The main components of walnut shells include acid-insoluble lignin, cellulose and hemicellulose. Although differences are found in the surface composition of walnut shells in different varieties and regions, the content of acid-insoluble lignin present in walnut 
shells is relatively high, accounting for more than $50 \%$ of the components. As the basic structure of the acid-insoluble lignin in the walnut shell exhibit mainly hydroxyl groups, phenolic groups, $\alpha-\mathrm{C}$ and $\gamma-\mathrm{C}$, that confer high chemical activity to the walnut shell. The introduction of hydrophilic groups at these sites with higher chemical activity gives the walnut shell filter material the ability to resist oil stain adhesion and easy backwash regeneration. Lignin has three structural units, namely, guaiacyl (G type), syringyl (S type) and hydroxyl-phenyl (H type), whereas the walnut shell acid-insoluble lignin mainly includes $\mathrm{G}$ and $\mathrm{S}$ types. The sulphite constant temperature reaction can be used to modify the lignin on the surface of the walnut shell; it is divided into three stages: soaking, sulfonating and dissolving [6-9]. To avoid serious damage to the lignin structure or dissolution of lignin during modification, the factors affecting the modification reaction (reaction temperature, drug mass fraction and reaction time) were optimised. The chemical reaction process is represented at Fig. S1.

\subsection{Filter Material Characterisation and Mechanical Property Analysis}

3.2.1. Analysis of the surface morphology of the filter material SEM (Fig. S2) results show that at different magnifications, the filter material surface before modification was oleophilic and had large and numerous micropores, thereby easily adsorbing emulsified oil and blocking the micropores. The filter material surface after modification became dense and had small micropores, thereby easily forming a trapping layer that prevented the blocking of micropores. In addition, the pollutant catching capacity of the modified filter material was easily restored by backwashing.

\subsubsection{EDS analysis}

Table 3 shows the EDS results of the filter material surface. The unmodified walnut shell contains four elements: C, O, Ca and $\mathrm{K}$. In addition to these elements, the modified walnut shell also has $\mathrm{Na}, \mathrm{S}$ and $\mathrm{Mg}$, it is indicated that the surface of the Walnut shells successfully introduces a hydrophilic sulfonic acid group.

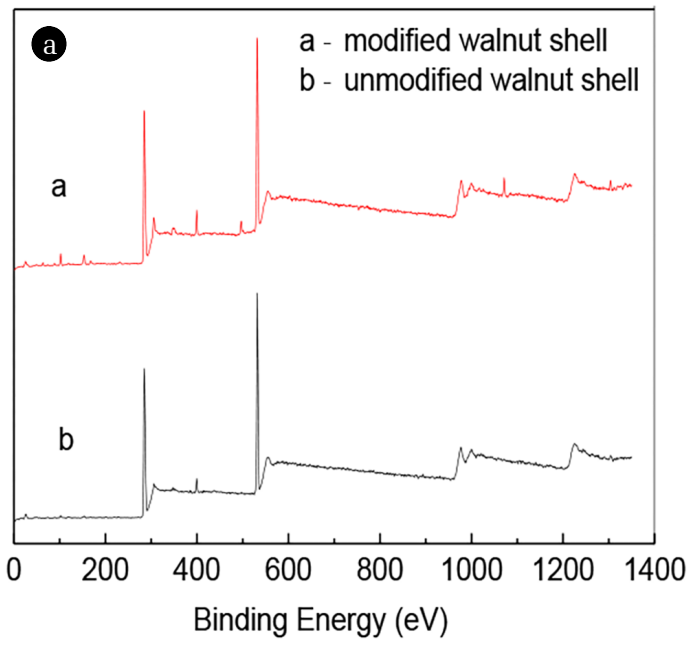

Fig. 4. XPS spectrum of walnut shell before and after modification.
Table 3. Walnut Shell Filter Material EDS Analysis (Raw and Modified Walnut Shell)

\begin{tabular}{|c|c|c|}
\hline Element & $\begin{array}{l}\text { Element content of } \\
\text { raw walnut shell/\% }\end{array}$ & $\begin{array}{l}\text { Element content of } \\
\text { modified walnut shell/\% }\end{array}$ \\
\hline $\mathrm{C}$ & 58.73 & 49.03 \\
\hline $\mathrm{O}$ & 32.11 & 25.96 \\
\hline $\mathrm{Au}$ & 5.94 & 9.02 \\
\hline $\mathrm{Na}$ & -- & 5.69 \\
\hline S & -- & 3.85 \\
\hline $\mathrm{Ca}$ & 2.03 & 3.06 \\
\hline $\mathrm{Si}$ & -- & 1.92 \\
\hline $\mathrm{Mg}$ & -- & 1.47 \\
\hline $\mathrm{K}$ & 1.2 & -- \\
\hline
\end{tabular}

\subsubsection{XPS analysis}

The XPS test (Fig. 4) shows that the unmodified walnut shell consists mainly of $\mathrm{O}$ (binding energy of $532.6 \mathrm{eV}$ ) and $\mathrm{C}$ (binding energy of $284.92 \mathrm{eV}$ ), with element contents of $27.43 \%$ and $67.77 \%$, respectively. After hydrophilic modification, $\mathrm{O}$ (binding energy of $532.77 \mathrm{eV}$ ) and $\mathrm{C}$ (binding energy of $286.21 \mathrm{eV}$ ) are considered main elements but their contents are changed. The content of $\mathrm{O}$ is increased to $29.68 \%$, and that of $\mathrm{C}$ is decreased to $65.14 \%$. The binding energies of the two elements are also changed. The peak binding energy of $\mathrm{O}$ is increased by $0.17 \mathrm{eV}$, and a new peak appears at $500 \mathrm{eV}$. The peak binding energy of $\mathrm{C}$ is increased by $1.29 \mathrm{eV}$, indicating that the chemical changes of the surface group of the walnut shell have occurred. The increase in the content of $\mathrm{O}$ element is due to the introduction of sulphonic acid group after sulphonation.

A significant $\mathrm{S}$ binding energy peak $(168 \mathrm{eV})$ is not observed on the XPS wide scan spectrum of the modified walnut shell due to the high content of $\mathrm{C}$ and $\mathrm{O}$ in the walnut shell and the limited introduction of sulfonic acid groups. The comparative results before and after modification show that on almost no $\mathrm{S}$ element is found on the surface of the unmodified walnut shell, and the hydrophilic sulphonic acid group is introduced into the surface of the modified walnut shell.

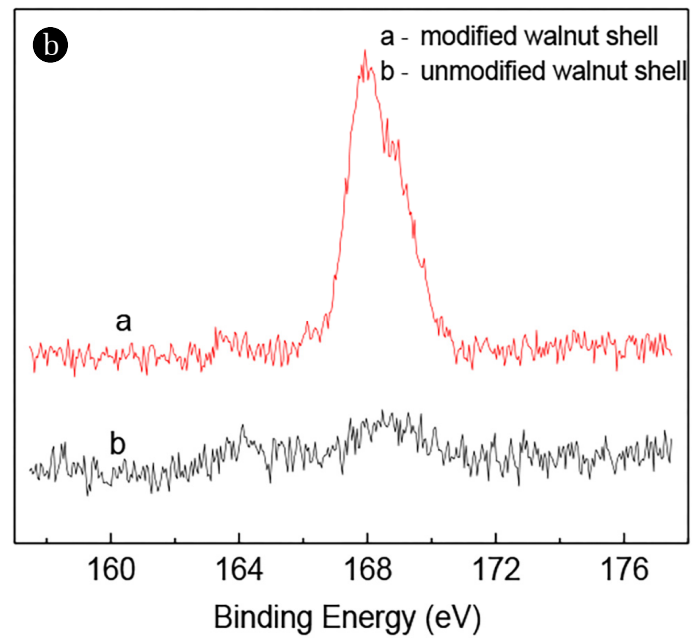

(a) Wide scan spectrum. (b) Narrow scan spectrum. 


\subsubsection{Evaluation of the surface wettability of filter materials} The contact angle $(\theta)$ is an important measure of the relationship between the material and the wettability of the liquid. $\Theta=90^{\circ}$ can be used as the boundary between wetting and nonwetting. When $\theta<90^{\circ}$, it can be wetted; when $\theta>90^{\circ}$, it cannot be wetted [10-13]. Table 4 shows that the contact angle of the unmodified walnut shell filter material is $97^{\circ}$, and the water droplets are rounded on the filter material surface, indicating that the surface is hydrophobic. The water droplets are spread on the surface of the modified walnut shell filter material, and the contact angles are reduced to $36.5^{\circ}-66.25^{\circ}$. The results indicate that the surface wettability of the modified walnut shell is significantly improved, and the surface becomes hydrophilic and oleophobic. The hydrophilic modification degree of the No. 1 filter material is the highest, and that of the No. 3 filter material is the lowest.

Therefore, the optimum conditions of the filter reaction, the ratio of the ratio of the agent to the filter material are: $\mathrm{Na}_{2} \mathrm{CO}_{3}$ $20.00 \%, \mathrm{NaHSO}_{3} 10.00 \%$ and $\mathrm{Na}_{2} \mathrm{SO}_{3} 0.10 \%$, and the reaction temperature is $105^{\circ} \mathrm{C}$ reflux reaction time $3 \mathrm{~h}$.

Table 4. Contact Angle of Different Walnut Shell Filter Materials $\left(^{\circ}\right)$

\begin{tabular}{lccc}
\hline Serial number & Once & Twice & Average value \\
\hline original filter material 0 & 93 & 97 & 95 \\
modified filter material 1 & 64 & 68.5 & 66.25 \\
modified filter material 2 & 66 & 66.5 & 66.25 \\
modified filter material 3 & 36.5 & 37 & 36.75 \\
modified filter material 4 & 56.5 & 55.5 & 56 \\
modified filter material 5 & 56.5 & 56 & 56.25 \\
modified filter material 6 & 47 & 44 & 45.5 \\
\hline
\end{tabular}

\subsubsection{Analysis of the mechanical property of filter materials}

Fig. S3 shows the stress-strain curves of Nos. 0 and 3. The two filter materials have a slight deformation of $0.01 \mathrm{~mm}$ at a force of $0.050 \mathrm{kN}$. When the destructive force reaches 50.00 $\mathrm{kN}$ with a force increase speed of $0.10 \mathrm{kN} / \mathrm{s}$ and the breaking strength is $39.80 \mathrm{MPa}$, the strains of Nos. 0 and 3 are $59.85 \%$ and $60.76 \%$, respectively. No comminute changes in the two samples are found, indicating that the mechanical properties of the walnut shells before and after modification are unchanged, and they still have the advantages of high hardness and good wear resistance.

\subsection{Effect of Filter Material on Filtration Oil Removal and Backwashing}

\subsubsection{Filtration oil removal effect}

Continuous dynamic degreasing experiment was conducted on No. 0, 1 and 3 filter materials. Fig. 2 and Fig. 3 show the degreasing rate and filtration time of the series of experiments, respectively. The emulsified and suspended oils are affected by various forces during filtration, including gravity sedimentation, hydraulic collision and Brownian diffusion during migration. The contact flocculation, electrostatic attraction, adsorption, molecular attraction and coalescence effect during adsorption affect the filtration and degreasing efficiency $[14,15]$. Fig. 2 shows that the average oil removal rates of Nos. 0, 1 and 3 are 38.05\%, 55.26\% and 89.51\%, respectively.
The degreasing rates of No. 1 and 3 modified filter materials are improved, and the degreasing effect of No. 3 is the best. This finding is caused by change in the surface properties of the modified walnut shell from lipophilic to hydrophilic and from oil wettability to water wettability during filtration. The contact angle between the oil granule and walnut shell in the water is changed from immersion adsorption to oil bead particle adsorption. Therefore, the contact area of the emulsified oil with the surface of the walnut shell is reduced, and the total amount of adsorption increased. The adsorption force of the oil beads follows the principle of chemical flotation kinetics $(F=4 \pi \mathrm{r} \sigma)$, wherein a large particle size of the oil bead shows a strong adhesion. Moreover, the large particle size of the oil beads has a high strength of coalescence. Fig. 3 shows that the average filtration time of No. 3 is $9.28 \mathrm{~min}$ and that of other filter materials is between $3.6 \mathrm{~min}$ and $5.1 \mathrm{~min}$. The filtration time of No. 3 is considerably larger than that of other filter materials. The reason is that in addition to the resistance of the filter layer formed by the walnut shell intercepting the emulsified oil and the blocking effect of the suspended particles, the surface of the modified walnut shell compared with the unmodified filter material has additional capillary resistance due to water wettability [16-18]. Thus, the filtration speed becomes slow, and the filtration time becomes long. Therefore, the emulsified oil in the water is in contact with the filter material for a long period, and its adsorption, retention and capture effects are enhanced.

\subsubsection{Filter backwashing evaluation}

Table S1 displays that the oil content of the backwashed effluent of No. 3 reaches the highest value of $405.15 \mathrm{mg} / \mathrm{L}$, indicating that the oil adsorbed by No. 3 is easily removed by backwashing, and its backwashing effect is greatly improved compared with those of the other materials. The surface of the modified walnut shell becomes hydrophilic and oleophobic. Thus, oil concentrates in the gap of the filter layer in the form of oil droplets; it is easily backwashed under water flushing. No. 3 captures a large amount of oil during filtration; thus, its backwashed effluent contains a large amount of emulsified oil.

\section{Conclusions}

The walnut shell filter was modified by sulphite reflux reaction under alkaline conditions. The best modification conditions were as follows: mass ratio $\left(\mathrm{m}_{\mathrm{x}} / \mathrm{m}_{1} \%\right) \mathrm{Na}_{2} \mathrm{CO}_{3} 20.00 \%, \mathrm{NaHSO}_{3} 10.00 \%$ and $\mathrm{Na}_{2} \mathrm{SO}_{3} 0.10 \%$, the reaction temperature is $105^{\circ} \mathrm{C}$ reflux reaction time $3 \mathrm{~h}$. The surface wettability of the walnut shell filter material before and after modification reversed from lipophilic to hydrophilic. For the treatment of polymer $(50.00 \mathrm{mg} / \mathrm{L})$ and oil (150.00-230.00 mg/L) containing wastewater, the degreasing rate of modified filter material was greater than $88 \%$, which was increased by more than $50.00 \%$ compared with that of the filter material before modification. The oil content in the backwashed effluent of modified filter material was $405.15 \mathrm{mg} / \mathrm{L}$ while in the oral filter material backwashed effluent was $100 \mathrm{mg} / \mathrm{L}$, indicating that the backwashing degreasing effect was also greatly improved. 


\section{Acknowledgment}

The authors are grateful for financial support from the National Science and Technology Major Project of China (No.2016ZX05025003).

\section{Author contributions}

X.Y. (Professor) conducted all the experiments. J.Z. (Ph.D. \& Chief Engineer) performed the experiments. X.J.W. (Ph.D.) performed the experiments. M.J.Z. (Ph.D.) wrote the manuscript.

\section{References}

1. Zhu MJ, Yao J, Wang WB, Yin XQ, Chen W, Wu XY. Using response surface methodology to evaluate electrocoagulation in the pretreatment of produced water from polymer-flooding well of Dagang Oilfield with bipolar aluminum electrodes. Desalin. Water Treat. 2016;57:15314-15325.

2. Dong XG, Liu SM, Yu ZC, Sun B, Niu YL. Experimental study on the filtration of wastewater containing polymer by axial dynamic backwashing filter. Ind. Water Treat. 2015;35:86-89 (in Chinese).

3. Srinivasan A, Viraraghavan T. Removal of oil by walnut shell media. Bioresour. Technol. 2008;99:8217-8220.

4. Wang JC, Du ZH, Zhao JT, Liu CM. A study on the recycling of walnut filter material by cleaning. Adv. Mater. Res. 2012;518-523:3267-3270.

5. Liu A, Liu S. Study on performance of three backwashing modes of filtration media for oilfield wastewater filter. Desalin. Water Treat. 2016;57:10498-10505.

6. Yang Y, Zhang X, Wang Z. Oilfield produced water treatment with surface-modified fiber ball media filtration. Water Sci. Technol. 2002;46:165-170.

7. Bao CX, Wei BG, Chang Q. Silane coupling agent for surface modification of quartz sand filter medium. China Environ. Sci. 2013;33:848-853 (in Chinese).

8. Zhu MJ, Liu RP, Wu XY, et al. Adsorption of petroleum hydrocarbon by modified walnut shell. Environ. Eng. 2015;33:54-58 (in Chinese).

9. Zhu MJ, Yao J, Dong LF, Sun JJ. Adsorption of naphthalene from aqueous solution onto fatty acid modified walnut shells. Chemosphere 2016;144:1639-1645.

10. Kord B. Studies on mechanical characterization and water resistance of glass fiber/thermoplastic polymer bionanocomposites. J. Appl. Polym. Sci. 2012;123:2391-2396.

11. Mandal A, Kar S, Kumar S. Synergistic effect of mixed surfactant (Tween 80 and SDBS) on wettability alteration of oil wet quartz surface. J. Dispersion Sci. Technol. 2016;37:1268-1276.

12. Yang BW, Chang Q. Wettability studies of filter media using capillary rise test. Sep. Purif. Technol. 2008;60:335-340.

13. Ortiz-Arroyo A, Larachi F, Iliuta I. Method for inferring contact angle and for correlating static liquid hold-up in packed beds. Chem. Eng. Sci. 2003;58:2835-2855.

14. Luo Y, Huang JG. Surface modification of natural cellulose substances: toward functional materials and applications. Sci. China Ser. B 2014;57:1672-1682 (in Chinese).

15. Liu G, Wei BG, Wu FP. Hydrophobic oil filter prepared by dry surface modification of quartz sand. J. Chem. Ind. Eng. 2016;67:2101-2108 (in Chinese).

16. Kang H, Zhao B, Li L, Zhang J. Durable superhydrophobic glass wool@polydopamine@ PDMS for highly efficient oil/water separation. J. Colloid Interface Sci. 2019;544:257-265.

17. Wang X, Li M, Shen Y, Yang Y, Feng H, Li J. Facile preparation of loess-coated membranes for multifunctional surfactant-stabilized oil-in-water emulsion separation. Green Chem. 2019;21:3190-3199

18. Yin X, Wang Z, Shen Y, Mu P, Zhu G, Li J. Facile fabrication of superhydrophobic copper hydroxide coated mesh for effective separation of water-in-oil. Sep. Purif. Technol. 2020;230: 115856-115862. 